\title{
PREVALENCIA Y FACTORES DE RIESGO RELACIONADOS CON LA CETOSIS CLÍNICA Y SUBCLÍNICA TIPO I Y II EN UN HATO DE VACAS JERSEY EN COSTA RICA
}

\author{
Alejandro Saborío-Montero ${ }^{1 / *}$, Jorge Ml. Sánchez*
}

\begin{abstract}
Palabras clave: Vacas lecheras, cetosis, condición corporal, ácido $\beta$-hidroxibutírico.
Keywords: Dairy cows, ketosis, body condition score, $\beta$-hidroxibutiric acid.
\end{abstract}

Recibido: $16 / 11 / 12$

\section{RESUMEN}

El objetivo de este estudio fue analizar la prevalencia y grado de cetosis tipo I y tipo II e investigar los factores de riesgo asociados con esta enfermedad metabólica, en un hato Jersey de 203 vacas en Oreamuno, Cartago, Costa Rica $\left(9^{\circ}\right.$ 55' Latitud Norte, $83^{\circ}$ 51' Longitud Oeste, 2350 $\mathrm{m}$ de altitud), para proponer prácticas de manejo y alimentación que contribuyan a reducir la incidencia de este desbalance metabólico. La prevalencia de cetosis tipo II y tipo I fue determinada midiendo la concentración sanguínea del ácido $\beta$-hidroxibutírico ( $\beta \mathrm{HBA}$ ) a los $8 \pm 3$ y $30 \pm 3$ días de lactancia en 117 y 114 animales, respectivamente. La cetosis clínica tipo II no fue detectada y $4,27 \%$ de las vacas tuvieron cetosis subclínica $\left(1,4\right.$ a 2,9 mmol..$\left.^{-1}\right)$ de este tipo. Los porcentajes de vacas con cetosis clínica $\left(>2,9\right.$ mmol. l $\left.^{-1}\right)$ y subclínica tipo I fueron 3,51 y 9,65 respectivamente. Durante la última semana de gestación, la pérdida de condición corporal difirió $(\mathrm{p}<0,05)$ para vacas sanas y cetóticas tipo I y fue de 0,09 y 0,31 puntos, respectivamente. Las vacas con cetosis tipo I fueron de mayor $(p<0,01)$ número de partos, duración del período seco más extensa $(\mathrm{p}<0,05)$ y mayor pico de lactancia $(\mathrm{p}<0,01)$,

1 Autor para correspondencia. Correo electrónico: alejandro.saboriomontero@ucr.ac.cr
Aceptado: 04/03/13
Prevalence and risk factors related with
clinical and subclinical ketosis type I and II in
a Jersey cow herd in Costa Rica. The aim of this study was to analyze the prevalence and grade of ketosis type I and II, and to examine risk factors related with this metabolic disease, in a 203 cows Jersey herd in Oreamuno, Cartago, Costa Rica ( $9^{\circ}$ $55^{\prime}$ North Latitude, $83^{\circ} 51^{\prime}$ West Longitude, 2350 $\mathrm{m}$ of altitude), to propose management and feeding practices that contribute to reduce de incidence of this metabolic imbalance. Prevalence of type II and type I ketosis was determined by measuring blood concentration of $\beta$-hydroxybutyric acid ( $\beta$ HBA) at $8 \pm 3$ and $30 \pm 3$ days in milk in 117 and in 114 animals, respectively. No clinical type II ketosis was detected, and $4.27 \%$ of the cows had subclinical (1.4 to $2.9 \mathrm{mmol}^{-1}$ ) ketosis of this type. Percentages of cows with clinical (>2.9 mmol. $1^{-1}$ ) and subclinical type I ketosis were 3.51 and 9.65, respectively. During the last week of gestation, body condition loss differed $(\mathrm{p}<0.05)$ for healthy and type I ketotic cows and was 0.09 and 0.31 points, respectively. Cows with type I ketosis were of greater $(\mathrm{p}<0.01)$ parity, longer $(\mathrm{p}<0.05)$ dry period length and greater $(\mathrm{p}<0.01)$
Centro de Investigaciones en Nutrición Animal y Escuela de Zootecnia, Universidad de Costa Rica. San José, Costa Rica. 
que las vacas sanas. Los resultados sugieren que calificar la condición corporal durante la última semana de gestación podría ser útil para predecir el riesgo de los animales a desarrollar cetosis tipo I. Basados en estos resultados, el manejo para evitar periodos secos mayores de 60 días ayudaría a reducir la incidencia de cetosis. Además, la alimentación y manejo de las vacas multíparas y vacas de mayor producción, conducente a reducir la pérdida de condición corporal post parto, también podrían reducir la incidencia de los diferentes tipos de cetosis.

\section{INTRODUCCIÓN}

La vaca lechera sufre cambios fisiológicos, metabólicos y sociales importantes durante la transición del período seco al de lactación, los cuales incrementan sustancialmente el riesgo de sufrir desbalances metabólicos o enfermedades de la producción tales como cetosis, hígado graso y desplazamiento del abomaso. La prevalencia de estas enfermedades se asocian a cambios abruptos en el metabolismo energético cuando la vaca pasa del estado de gestación en que requiere cantidades moderadas de energía para el desarrollo fetal, a un estado de lactancia en que las necesidades de energía se triplican para sustentar primero la síntesis de calostro y luego la de leche. Cuando la vaca no responde en forma apropiada a este desbalance de energía la relación entre la hormona del crecimiento y la insulina en sangre promueve una movilización de ácidos grasos de cadena larga del tejido adiposo. Cuando la concentración de ácidos grasos no esterificados en la sangre sobrepasa la capacidad oxidativa del hígado se produce una acumulación de ácidos grasos en este órgano, lo mismo que la producción de cuerpos cetónicos (acetoacetato y $\beta$ HBA), lo que causa la cetosis clínica o subclínica (Drackley 1997, Duffield 2000, Epperson 2005, NRC 2001).

La cetosis se puede dar en forma clínica o subclínica, siendo la clínica aquella que se peak milk yield, than healthy cows. Results suggest that scoring body condition during the last week of gestation could be useful to predict the risk of the animals developing type I ketosis. Based on these results, management to avoid dry periods in excess of 60 days would help to reduce the incidence of ketosis. Furthermore, feeding and management practices of older and higher producing cows, to reduce the loss of body condition post calving, could also reduce the incidence of ketosis.

caracteriza por la aparición repentina de signos como pérdida del apetito, particularmente del alimento balanceado, disminución de la producción de leche, rápida pérdida de condición corporal (Baird 1982) y constipación (Johnson y Otterby 1981). La cetosis sub clínica se da cuando hay elevada concentración de cuerpos cetónicos circulantes en la ausencia de signos clínicos (Andersson 1988).

La cetosis espontánea o de subalimentación es la forma clásica de cetosis que ocurre en vacas entre las 3 y 6 semanas postparto. Ésta es llamada cetosis tipo I por sus similitudes con la diabetes mellitus tipo I. En ambas condiciones, la concentración de insulina en sangre es baja, aunque por distintas razones. La insulina es baja en la diabetes tipo I debido a defectos pancreáticos, mientras que en la cetosis tipo I, la insulina es baja debido a una hipoglucemia crónica por falta de precursores de glucosa (Oetzel 2007). La cetosis tipo II incluye la vieja designación de "síndrome de la vaca gorda". Esta incluye cualquier vaca que desarrolle un balance energético negativo e inicie la movilización de grasa del cuerpo antes o durante el parto. Las vacas gordas están en mayor riesgo de sufrir este desbalance debido a que ellas son más propensas a deprimir el consumo de materia seca cerca del parto (Treacher et ál. 1986), pero las vacas flacas también son susceptibles si el manejo nutricional durante el periodo de 
transición es inadecuado (Oetzel 2007). Ambos tipos de cetosis pueden presentar sintomatología clínica; de igual manera, la forma subclínica de la enfermedad puede presentarse indistintamente del tipo de cetosis.

La incidencia de la cetosis clínica es de alrededor de $6 \%$ y la subclínica de $40 \%$ en hatos confinados. Esta enfermedad causa reducciones importantes en la producción de leche y sus componentes en las vacas que la sufren, así como una reducción de la vida productiva dentro del hato, incrementos en la incidencia de otras enfermedades de la producción y problemas reproductivos. Lo anterior hace que la prevalencia de esta enfermedad sea muy onerosa para la economía de los hatos de ganado lechero (Drackley 1997, Duffield 2000, Epperson 2005, NRC 2001).

Debido a que las prácticas de manejo y alimentación de los hatos lecheros en ambientes estabulados; hatos en los que se ha realizado la mayoría de los estudios sobre esta enfermedad; difieren de los sistemas de pastoreo en ambientes tropicales, se considera importante analizar los determinantes involucrados en el desarrollo de este padecimiento. El objetivo de esta investigación fue identificar los factores de riesgo asociados a la prevalencia de la cetosis en un hato de ganado Jersey en pastoreo, a fin de ofrecer un modelo de abordaje metodológico para el estudio de esta enfermedad en hatos individuales en ambientes tropicales.

\section{MATERIALES Y MÉTODOS}

La investigación se realizó en una finca comercial de ganado lechero ubicada en el cantón de Oreamuno a $9^{\circ} 55^{\prime}$ latitud Norte, $83^{\circ} 51^{\prime}$ longitud Oeste y a una altitud de 2350 msnm, en Cartago, Costa Rica. La temperatura y precipitación media anual en la zona fueron de $17,5^{\circ} \mathrm{C}$ y $1800 \mathrm{~mm}$, respectivamente (Costa Rica, IMN 2012). El hato constó de 203 vacas de la raza Jersey y fueron evaluadas un total de 122 vacas cuyo número de partos osciló entre 1 y 10, las cuales parieron en el período comprendido entre el 3 de setiembre de 2010 y el 23 de abril de 2011.
La alimentación de todos los animales se basó en el uso intensivo del pasto kikuyo (Kikuyuocloa clandestina), pastoreado a 30 días de rebrote y que en promedio tuvo 16,4\% M.S., $21,5 \%$ P.C., $60,5 \%$ F.D.N., $28,5 \%$ F.D.A., 3,1\% de lignina, 1,3 Mcal de $\mathrm{EN}_{\mathrm{L}} / \mathrm{kg}(3 \mathrm{X})$ y $0,35 \%$ de $\mathrm{Ca}$, de la MS. Las vacas secas (-9 a -4 semanas con respecto al parto) y las próximas al parto (-3 semanas al día de parición) pastorearon en secuencias de pastoreo diferentes a las vacas que estaban en lactación. Además del pasto, las vacas secas en promedio consumieron $2 \mathrm{~kg}$ de maíz por día. Así mismo, durante las 3 semanas previas al parto los animales consumieron 4 $\mathrm{kg}$ de un alimento formulado específicamente para este estado fisiológico (16\% PC, 1,7 Mcal de $\mathrm{EN}_{\mathrm{L}} / \mathrm{kg}(1 \mathrm{X}), 35 \%$ de almidón, 0,2\% de $\mathrm{Ca}$, de la MS) y al inicio de la lactancia se les suministró $4 \mathrm{~kg}$ de alimento para lactación (16\% PC, 1,9 Mcal de $\mathrm{EN}_{\mathrm{L}} / \mathrm{kg}$ (3X), 48\% de almidón, $0,9 \%$ de $\mathrm{Ca}$, de la MS), cantidad que fue incrementada paulatinamente según la producción de cada vaca. Cada animal también consumió diariamente en promedio $1 \mathrm{~kg}$ de heno (Digitaria decumbens Stent, cv. Transvala) (82,3\% M.S., 5,1\% P.C., 61,4\% F.D.N., 31,6\% F.D.A. 1,25 Mcal de $\mathrm{EN}_{\mathrm{L}} / \mathrm{kg}(1 \mathrm{X}), 2,3 \%$ Lignina, de la MS). El alimento balanceado se ofreció antes de cada uno de los 2 ordeños realizados por día. Los análisis de los pastos y alimentos balanceados se practicaron de acuerdo con las metodologías propuestas por el AOAC (2000), NRC (2001) y Van Soest et ál. (1991).

La medición de la concentración de $\beta \mathrm{HBA}$ en sangre entera se realizó con el medidor electroquímico de mano "Optium Xceed ${ }^{\circledR}$ " de Laboratorios Abbott, mediante la inmersión del dispositivo sensible desechable del equipo dentro del tubo de ensayo que contenía la sangre objeto de análisis. La muestra de sangre se obtuvo por punción de la vena y/o arteria coxígea con una aguja de tamaño $21 \times 1^{1 / 2}$ y un tubo estéril VACUTTE ${ }^{\circledR}$ de $9 \mathrm{ml}$ con activador de aglutinación de suero Z. De los 122 animales, 117 se sangraron en el día $8 \pm 3$ posparto y 114 en el día $30 \pm 3$ posparto. Períodos que se consideran 
de mayor riesgo para el desarrollo de la cetosis tipo II y tipo I, respectivamente (Gillund et ál. 2001, Juchem et ál. 2004, Nielsen et ál. 2005, Oetzel 2007). Debido a la muerte de un animal por causas diferentes a cetosis y a la pérdida de aretes de identificación en algunas vacas, no fue posible muestrear al mismo número de animales en ambos periodos.

El principio en que se basa el medidor electroquímico de mano, consiste en la oxidación del $\beta$ HBA en la muestra de sangre para producir acetoacetato, en presencia de la enzima hidroxibutirato deshidrogenasa, con la reducción de $\mathrm{NAD}^{+}$a NADH. Posteriormente el NADH es oxidado nuevamente a $\mathrm{NAD}^{+}$por un mediador redox. La corriente eléctrica generada en estas reacciones es directamente proporcional a la concentración de $\beta \mathrm{HBA}$ en la muestra que se analiza. Después de 10 segundos la concentración de $\beta$ HBA $\left(\mathrm{mmol}^{-1} \mathrm{l}^{-1}\right)$ es cuantificada y desplegada en el medidor electroquímico de mano (Iwersen et ál. 2009).

Los animales que presentaron concentraciones sanguíneas inferiores a $1,4 \mathrm{mmol}^{\mathrm{l}^{-1}}$ de $\beta$ HBA se consideraron negativos a cetosis. El diagnóstico de cetosis subclínica se obtuvo al presentarse valores entre 1,4 y $2,9 \mathrm{mmol}^{-1} \mathrm{l}^{-1}$ de $\beta$ HBA y la cetosis clínica cuando la concentración fue mayor a 2,9 mmol. $\mathrm{l}^{-1}$ (Oetzel 2007).

Duffield (2000), asevera que el estándar por excelencia para el diagnóstico de cetosis subclínica es la medición de $\beta$ HBA en suero o plasma sanguíneo. Según Iwersen et ál. (2009), al usar sangre entera y un valor umbral de $1400 \mu$ moles. $^{l^{-1}}$ de $\beta$ HBA de sangre, el medidor electrónico de mano para la medición de $\beta$ HBA en vacas lecheras (Precision Xtra ${ }^{\circledR}$, Abbott Diabetes Care, Abingdon, U.K.) alcanzó $96 \%$ de sensibilidad y $97 \%$ de especificidad en 926 muestras analizadas, el cual es un desempeño de diagnóstico mayor que el de otros test químicos comúnmente utilizados (Ketostix ${ }^{\circledR}$, $\operatorname{Ketolac}^{\circledR}$ ), para el diagnóstico de esta enfermedad. La pertinencia del uso del equipo Precision Xtra ${ }^{\circledR}$ de Abbott Diabetes Care, Abingdon, U.K., para el diagnóstico de cetosis también es sustentada por Oetzel y McGuirk (2010).
Una sola persona calificó semanalmente la condición corporal de los animales desde el momento del secado (9 semanas preparto) hasta el final de la lactancia, mediante la metodología descrita por Ferguson et ál. (1994). La producción de leche se midió por medio de medidores automáticos Waikato® y la información tanto productiva como reproductiva fue introducida por un único individuo de manera quincenal en el sistema de registros computarizados VAMPP Bovino 3.0.

Para el análisis estadístico de la información se utilizó el paquete estadístico SPSS $^{\circledR}$. Se agruparon las vacas que presentaron una concentración sanguínea de $\beta$ HBA menor a $1,4 \mathrm{mmol}^{-1} \mathrm{l}^{-1}$ en la categoría de sanas. Así mismo, los animales con cantidades mayores o igual a $1,4 \mathrm{mmol}^{-1} \mathrm{l}^{-1}$ de $\beta$ HBA se clasificaron como enfermos. Para estimar estadísticamente las diferencias entre categorías y entre periodos de muestreo se utilizó la prueba t de Student para las variables concentración de $\beta$ HBA sanguíneo, cambio en condición corporal preparto, número de parto, duración del periodo seco y producción de leche al pico. Además se construyó un modelo de regresión logística para obtener la razón de ventaja, por medio de la variable respuesta prevalencia de cetosis a los $30 \pm 3$ días posparto, donde las vacas se agruparon en sanas y enfermas, a través de los predictores de las variables número de parto y periodo seco.

\section{RESULTADOS Y DISCUSIÓN}

\section{Prevalencia de la enfermedad}

La prevalencia de cetosis según los diferentes tipos y grado de afectación de la enfermedad se presentan en el Cuadro 1. De los 114 animales evaluados a los 30 \pm 3 días, 99 tuvieron una concentración sanguínea de $\beta$ HBA inferior a 1,4 mmol. $^{-1}$ (promedio 0,69), 11 de 1,4 a 2,9 mmol. $1^{-1} \beta$ HBA (promedio 1,98 ) y 4 más de 2,9 mmol. $1^{-1}$ BHBA (promedio 3,60), lo que indica que la prevalencia de cetosis tipo I en su forma subclínica en el hato evaluado fue $9,65 \%$ y en la forma clínica $3,51 \%$. 
Cuadro 1. Prevalencia de cetosis según su tipo y grado de afectación en un hato Jersey en pastoreo.

\begin{tabular}{llccc}
\hline Tipo & Grado & Animales muestreados & Animales positivos & Prevalencia (\%) \\
\hline I & Subclínica & 114 & 11 & 9,65 \\
I & Clínica & 114 & 4 & 3,51 \\
II & Subclínica & 117 & 5 & 4,27 \\
II & Clínica & 117 & 0 & 0 \\
\hline
\end{tabular}

En cuanto a la prevalencia de cetosis tipo II, 112 animales estudiados tuvieron una concentración de $\beta$ HBA en sangre inferior a 1,4 mmol. $1^{-1}$ (promedio 0,61 ) a los $8 \pm 3$ postparto, 5 de 1,4 a 2,9 mmol. $\mathrm{l}^{-1}$ (promedio 1,90 ) y ninguno tuvo concentraciones de este cuerpo cetónico superiores a 2,9 mmol. $\mathrm{l}^{-1}$, por lo que la prevalencia de cetosis tipo II subclínica en el hato de 117 vacas estudiado fue de $4,27 \%$.

En hatos estabulados de la raza Holstein, Oetzel (2007) al evaluar estadísticas sobre la incidencia de cetosis en múltiples publicaciones científicas informa que la prevalencia promedio de esta enfermedad en su forma clínica es de alrededor del $15 \%$. El mismo autor sugiere utilizar el valor de $10 \%$ de prevalencia de cetosis clínica como el valor a partir del cual debe hacerse una revisión integral de las prácticas de manejo y alimentación del hato lechero para hacer las correcciones correspondientes. En relación con la incidencia de cetosis subclínica, Duffield (2003) advierte que la prevalencia de esta enfermedad en hatos estabulados frecuentemente es superior a $40 \%$, lo que aumenta la probabilidad de que los animales sufran diferentes enfermedades y tengan un desempeño productivo y reproductivo inferior al esperado. Lo anterior indica que la prevalencia de la cetosis en el hato Jersey estudiado y cuya alimentación se basó en el uso intensivo de forrajes es inferior al reportado por diferentes autores que han investigado esta enfermedad en hatos en su mayoría de la raza Holstein en estabulación.

Las concentraciones promedio de $\beta \mathrm{HBA}$ durante los periodos $8 \pm 3$ y $30 \pm 3$ días posparto fueron de 0,66 y $0,91 \mathrm{mmol.l^{-1 }}$, respectivamente (Figura 1). Los valores difieren de manera

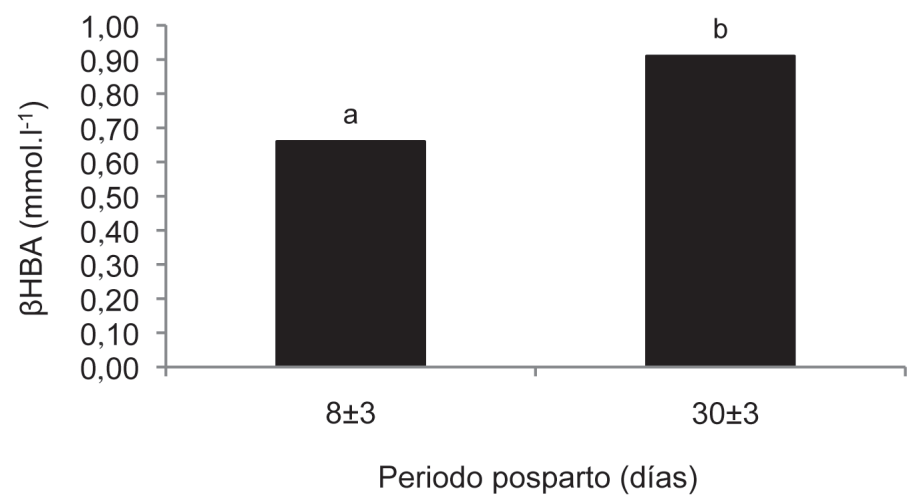

Barras con letras distintas implican diferencias altamente significativas $(\mathrm{p}<0,01)$ según la prueba t de Student para muestras apareadas.

Fig. 1. Concentración de BHBA según periodo de muestreo posparto, en vacas Jersey que consumen pastos tropicales. 
altamente significativa $(\mathrm{p}<0,01)$ entre sí, lo que sugiere que los animales en este hato son más afectados por una hipoglucemia crónica, a causa de una demanda no satisfecha de precursores de glucosa (cetosis tipo I), más que a una alta movilización de lípidos corporales debido a un balance energético negativo propio del inicio de la lactancia (cetosis tipo II). La mayor concentración de $\beta \mathrm{HBA}$ en sangre en el muestreo practicado a los $30 \pm 3$ días posparto indica que los animales movilizan más tejido adiposo en este período para tratar de lograr un balance energético (NRC 2001). Solamente $4,27 \%$ de los animales presentaron algún grado de cetosis en el periodo $8 \pm 3$ días posparto, mientras que $13,16 \%$ lo sufrieron en el periodo $30 \pm 3$ días posparto, lo que confirma la mayor propensión de este hato a cetosis tipo I.

La condición corporal promedio medida durante la primera semana postparto fue de 3,87; los valores medios de $\beta$ HBA obtenidos a los $8 \pm 3$ días son muy bajos, más si se considera que las vacas que llegan sobre acondicionadas al parto son más propensas a presentar cetosis (Gillund et ál. 2001). Esta situación podría deberse a una baja movilización de tejido subcutáneo durante este periodo, causado por el efecto de la raza y una producción de leche moderada al inicio de la lactación. French (2006) indica que los animales de la raza Jersey disminuyen en menor proporción el consumo de materia seca durante el periparto (17\%) con respecto a vacas de la raza Holstein $(35 \%)$. Lo anterior sugiere que las vacas de raza Jersey podrían depender menos de las reservas corporales durante el periodo de más alto riesgo a sufrir cetosis tipo II, que otras razas. Además Prendiville et ál. (2009) y Mackle et ál. (1996) indican que las vacas Jersey tienen una mayor capacidad de consumo de materia seca por unidad de peso vivo que aquellas de la raza Holstein, lo que podría explicar una propensión diferente de las razas a la prevalencia de esta enfermedad metabólica.

\section{Condición corporal}

La Figura 2 muestra el cambio de condición corporal durante la última semana preparto,

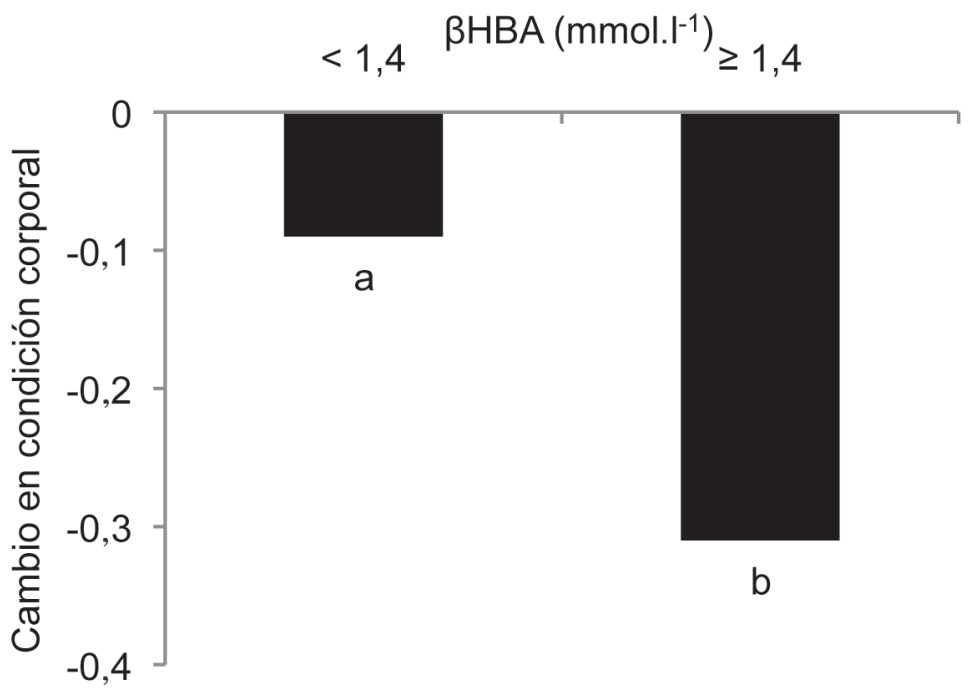

Barras con letras diferentes implican diferencias significativas $(\mathrm{p}<0,05)$ según la prueba t de Student para muestras independientes.

Fig. 2. Cambio de condición corporal en la última semana preparto según la concentración de $\beta$ HBA promedio a los $30 \pm 3$ días posparto para las categorías $<1,4 \mathrm{y} \geq 1,4 \mathrm{mmol.1^{-1 }}$, en un hato Jersey en pastoreo. 
esta fue significativamente $(\mathrm{p}<0,05)$ mayor en los animales que padecieron algún grado de cetosis a los $30 \pm 3$ días posteriores al parto (Promedio $-0,31$ ), que en animales sanos (Promedio -0,09), lo que indica que los animales que pierden más condición corporal durante esta semana son más propensos a presentar algún grado de cetosis tipo I.

La pérdida acelerada de condición corporal en este periodo previo al parto probablemente se deba a una disminución del consumo de materia seca. Grummer (1993) menciona que 3 semanas antes de la parición se dan reducciones graduales en el consumo de alimento, el cual se acelera durante los 7 días previos al parto. Hayirli et ál. (2002) encontraron que el consumo de materia seca decreció $32 \%$ durante las últimas 3 semanas de la gestación en vacas Holstein y que el $89 \%$ de ese decremento ocurrió durante la última semana de gestación.

El cambio en la condición corporal durante la última semana preparto podría ser utilizado como un indicador de la propensión de los animales a tener niveles altos de $\beta \mathrm{HBA}$, con el objetivo de modificar las prácticas de manejo y alimentación en el hato.

La condición corporal en la semana del parto no se relacionó con la prevalencia de cetosis en ninguno de los dos periodos de estudio, esto difiere de lo observado en otros estudios (Gearhart et ál. 1990, Gillund et ál. 2001) en los cuales hubo una relación entre la condición corporal al parto y la propensión a sufrir cetosis. Gillund et ál. (2001) señalan en su estudio que las vacas con cetosis perdieron significativamente más condición corporal en comparación con las vacas sanas durante la lactancia. Esta asociación tampoco fue encontrada en la presente investigación.

\section{Número de parto}

El coeficiente de correlación entre el número de parto y la concentración sanguínea de $\beta$ HBA a los $8 \pm 3$ y $30 \pm 3$ días posparto fue de 0,256 y 0,416 respectivamente, en ambos casos con un nivel alto de significancia estadística $(\mathrm{p}<0,01)$.

La Figura 3 denota la tendencia de la con-

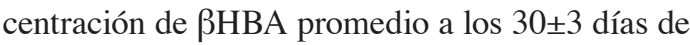
lactancia según el número de parto. Al agrupar los datos según los umbrales de concentración sanguínea de $\beta$ HBA a los $8 \pm 3$ días posparto (vacas sanas con concentraciones menores a 1,4 mmol. $^{-1}$ y enfermas con 1,4 mmol. $\mathrm{l}^{-1} \mathrm{o}$ más), se observa que al aumentar el número de parto promedio, aumenta la concentración de $\beta$ HBA sanguínea, que muestra diferencias significativas $(p<0,05)$ entre el número de parto promedio de las vacas sanas (promedio 3,21) y las enfermas (promedio 6,40). Estas diferencias también se observan al agrupar de la misma manera a los animales muestreados a los 30 3 días posparto, obteniéndose como número de parto promedio 3,05 para las vacas sanas y 5,13 para las enfermas (Figura 4).

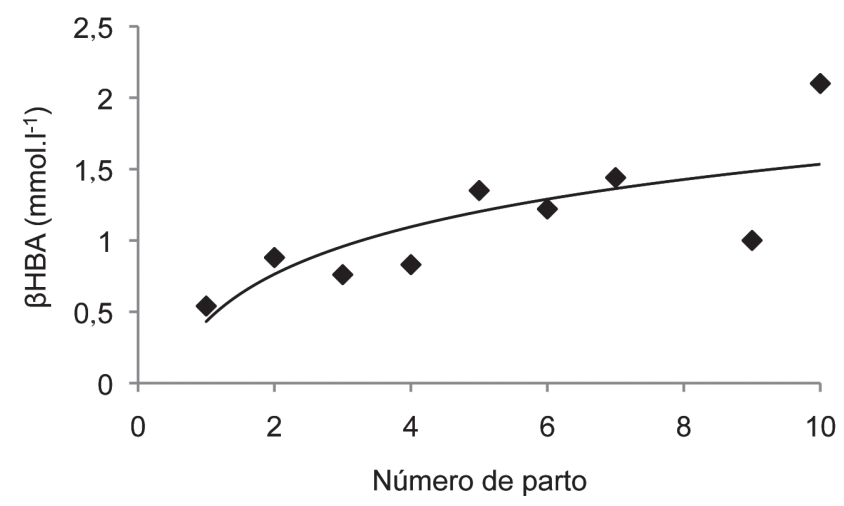

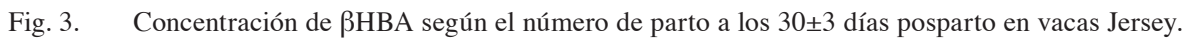




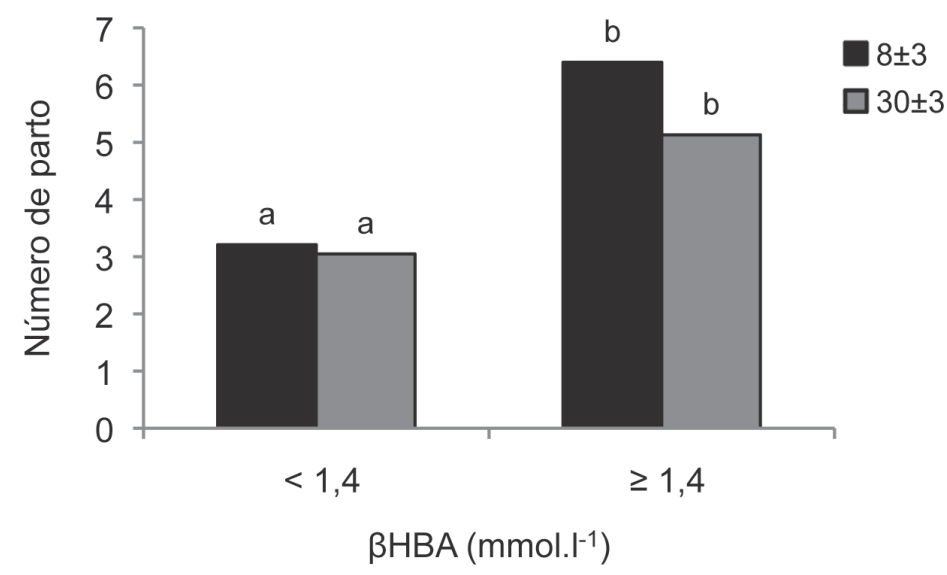

Barras con letras diferentes entre periodos de muestreo implican diferencias significativas $(\mathrm{p}<0,05)$ según la prueba t de Student para muestras independientes.

Fig. 4. Número de parto promedio según concentración de $\beta$ HBA para las categorías sanas $\left(<1,4\right.$ mmol.1 $\left.{ }^{-1}\right)$ y enfermas $(\geq 1,4$ mmol. $\left.1^{-1}\right)$ a los $8 \pm 3$ y $30 \pm 3$ días posparto en vacas Jersey que pastorean forrajes tropicales.

Al utilizar el modelo de regresión logística, la variable respuesta prevalencia de cetosis a los $30 \pm 3$ días posparto y como predictor la variable número de parto, indica que por cada incremento en una unidad de número de parto, se aumenta en 1,38 veces $(\mathrm{p}<0,01)$ la probabilidad de que una vaca presente cetosis en este periodo (Figura 5).

La información obtenida indica que al aumentar el número de parto, aumenta la concentración de $\beta$ HBA posparto en ambos periodos, por ende entre mayor sea el número de parto de una vaca, mayor será su propensión a sufrir cetosis tipo I y tipo II. Bobe et ál. (2004) mencionan en una extensa revisión de literatura que hay una asociación fuerte entre la concentración de $\beta$ HBA en el plasma sanguíneo y el número de parto. Lo que concuerda con lo encontrado en el presente estudio. Al mismo tiempo Bobe et

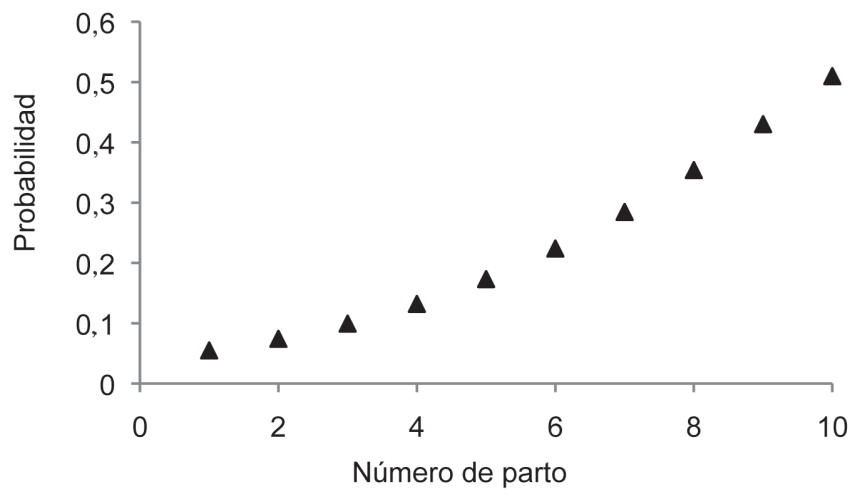

Fig. 5. Razón de ventaja de sufrir cetosis conforme aumenta el número de parto, según concentración de $\beta$ HBA promedio

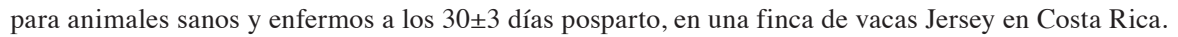


ál. (2004) menciona que la mayor proporción de vacas con hígado graso son aquellas más viejas.

\section{Periodo seco}

Al correlacionar el periodo seco de las vacas con la concentración de $\beta$ HBA a los $30 \pm 3$ días posparto se encontró una asociación significativa, el coeficiente de correlación obtenido fue de $0,235(\mathrm{p}<0,05)$. Para el periodo $8 \pm 3$ días posparto no se encontró esta asociación.

Watters et ál. (2008) indican que solamente 2 estudios han evaluado el efecto del periodo seco sobre las tasas de incidencia de enfermedades metabólicas. Estos autores encontraron que las vacas asignadas a un periodo seco de 34 días presentaron menor concentración de ácidos grasos no esterificados (NEFA) posparto, que aquellas asignadas a un periodo seco de 55 días. Sin embargo, la incidencia de cetosis no difirió entre tratamientos. El otro estudio realizado por Coppock et ál. 1974 menciona que periodos secos de 20, 30, 40, 50 y 60 días afectaron la incidencia de cetosis, al ser mayor en los períodos más largos.
Al agrupar a las vacas como sanas $(<1,4$ mmol. $\left.\mathrm{l}^{-1}\right)$ y enfermas $\left(\geq 1,4 \mathrm{mmol. \textrm {l } ^ { - 1 } )}\right.$ según la concentración de $\beta$ HBA a los $30 \pm 3$ días posparto, se encontraron diferencias $(p<0,05)$ entre grupos (Figura 6), donde los animales que presentaron algún grado de cetosis tuvieron un periodo seco más prolongado (13,1 días de más) que aquellos sanos. Los periodos secos promedio para las vacas con algún grado de cetosis y las sanas fueron de 78,3 y 65,2 días, respectivamente.

La prevalencia de cetosis tipo I para animales con un periodo seco de más de 60 días fue de $20,7 \%$, mientras que para animales con periodos secos inferiores al período clásico de 60 días fue de $7,1 \%$, esta información revela que la prolongación del periodo seco por encima de 60 días, se asocia con el aumento en la prevalencia de cetosis tipo I.

Al construir el modelo de regresión logística mediante la variable respuesta prevalencia de cetosis a los $30 \pm 3$ días posparto y como predictor la variable periodo seco, se obtuvo que vacas con un periodo seco de 1,2 y 3 semanas por encima de 60 días son 1,$21 ; 1,47$ y 1,79 veces más propensas $(p<0,05)$ respectivamente, a sufrir cetosis en

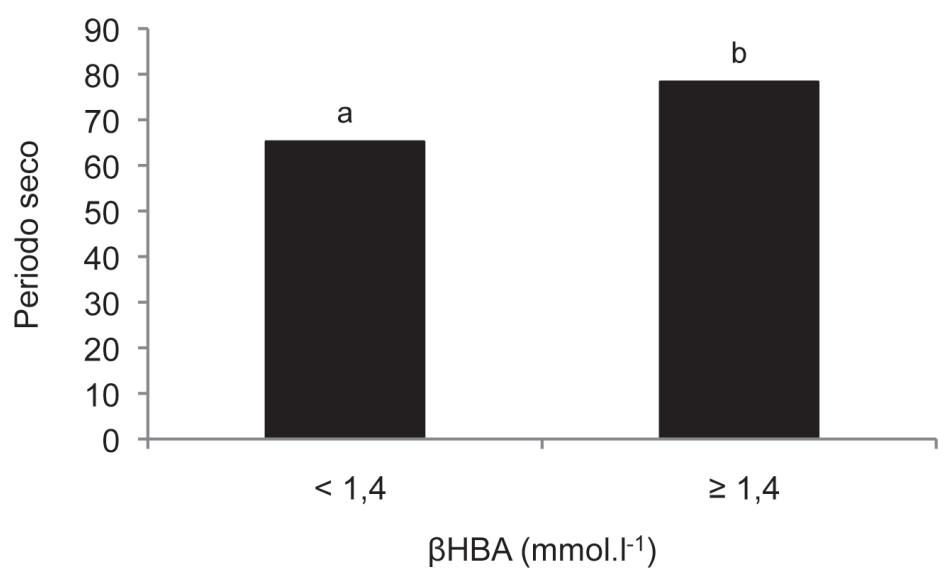

Barras con letras diferentes implican diferencias significativas $\mathrm{p}<0,05$ según la prueba t de Student para muestras independientes.

Fig. 6. Periodo seco según concentración de $\beta$ HBA para las categorías sanas $\left(<1,4\right.$ mmol. $\left.1^{-1}\right)$ y enfermas $\left(\geq 1,4\right.$ mmol.1 $\left.1^{-1}\right)$ a los $30 \pm 3$ días posparto en un hato de la raza Jersey. 
este periodo (Figura 7). Estos hallazgos sugieren que al aumentar el número de días que una vaca permanece seca, se incrementa la probabilidad de que la vaca tenga mayores concentraciones de $\beta$ HBA sanguíneo a los $30 \pm 3$ días posparto. Una posible explicación a estos hallazgos podría ser que al aumentar el número de días sin demandas fisiológicas de nutrientes para la producción láctea, se provoca una mayor acumulación de reservas corporales, y por consiguiente una mayor movilización de éstas previo al parto. Como anteriormente se expuso, dicho aumento en la movilización de reservas, está asociada a un aumento en la concentración de $\beta$ HBA sanguíneo a los $30 \pm 3$ días posparto y por lo tanto al aumento en la probabilidad de presentar cetosis en este periodo.

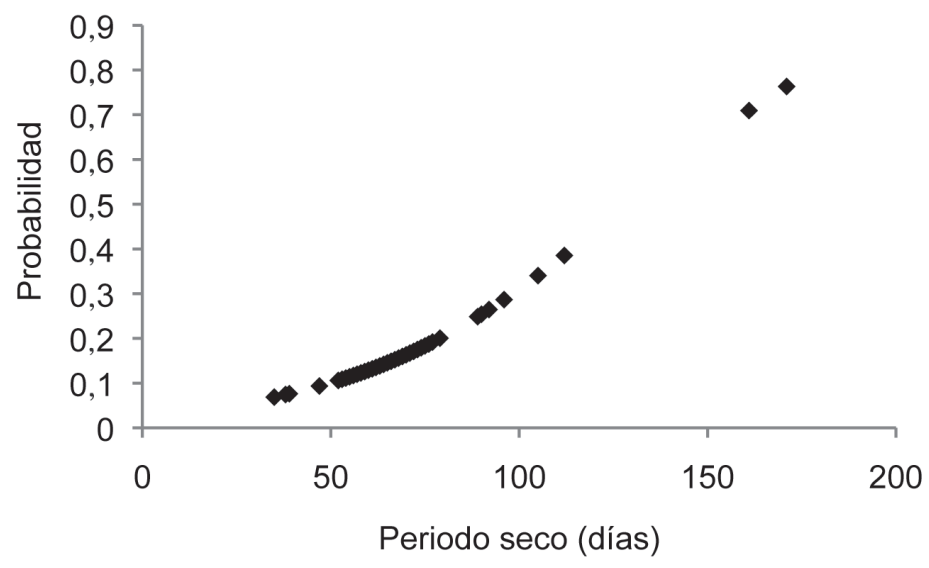

Fig. 7. Razón de ventaja de sufrir cetosis conforme aumenta el periodo seco, según concentración de $\beta$ HBA promedio a los $30 \pm 3$ días posparto, en vacas Jersey en Costa Rica.

\section{Producción de leche}

El coeficiente de correlación encontrado entre el pico de producción y la concentración de $\beta$ HBA a los $8 \pm 3$ días posparto no fue significativo; sin embargo, al correlacionar estas mismas variables en el periodo $30 \pm 3$ días posparto, se encontró un coeficiente de correlación de 0,304 ( $\mathrm{p}<0,01)$.

$\mathrm{Al}$ agrupar a las vacas como sanas $\left(\beta \mathrm{HBA}<1,4 \mathrm{mmol} . \mathrm{l}^{-1}\right)$ y con algún grado de cetosis $\left(\beta \mathrm{HBA} \geq 1,4 \mathrm{mmol} . \mathrm{l}^{-1}\right)$, se encontró que los valores del pico de producción de leche difieren significativamente $(\mathrm{p}<0,05)$ (Figura 8$)$, donde los animales con algún grado de cetosis tuvieron una mayor producción de leche (29,8kg.), en comparación con los animales considerados sanos (27,0 kg).
Lo anterior es contrario a lo esperado puesto que animales con mayor contenido de cuerpos cetónicos en sangre tienden a disminuir marcadamente el consumo de alimento balanceado y como consecuencia disminuyen su producción. Sin embargo debe mencionarse que del total de animales estudiados, únicamente uno $\left(\beta \mathrm{HBA}=3,1 \mathrm{mmol. \textrm {l } ^ { - 1 } )}\right.$ presentó síntomas clínicos de la enfermedad, al considerar una disminución evidente del consumo de alimento balanceado. Una posible explicación a lo observado podría ser que los animales de mayor número de parto en esta finca, fueron seleccionados por sus buenas características productivas y como se observa en la Figura 3, al aumentar el número de parto también aumenta la concentración de $\beta$ HBA, por lo que la asociación positiva entre producción de 


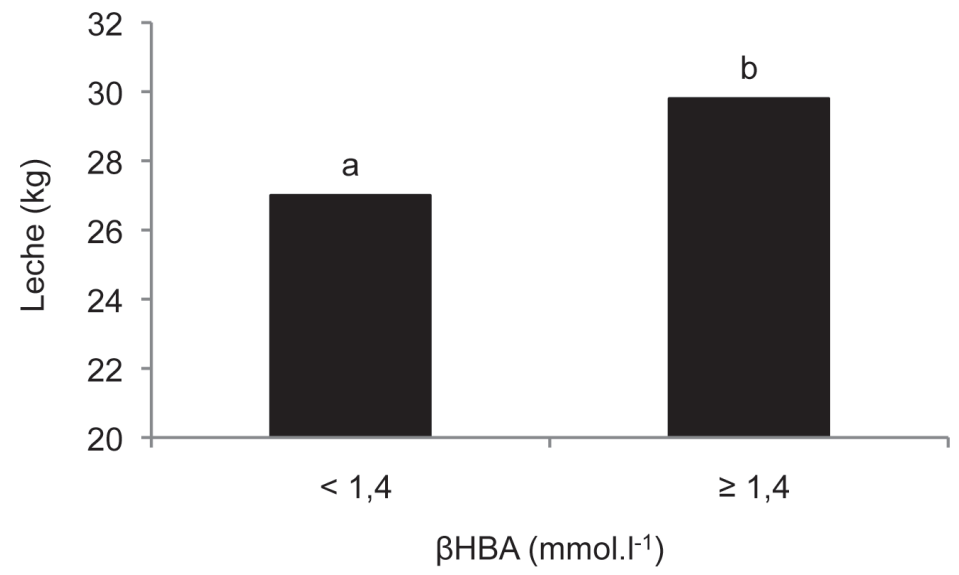

Barras con letras diferentes implican diferencias significativas $\mathrm{p}<0,05$ según la prueba t de Student para muestras independientes.

Fig. 8. Pico de producción de leche según concentración de $\beta$ HBA promedio para las categorías sanas $\left(<1,4\right.$ mmol.1 $\left.{ }^{-1}\right)$ y enfermas $\left(\geq 1,4 \mathrm{mmol}^{-\mathrm{l}^{-1}}\right)$ a los $30 \pm 3$ días posparto, en vacas Jersey que consumen pastos tropicales.

leche y concentración de $\beta$ HBA podría estar confundida por otros factores de mayor relevancia como el número de parto.

Otra posible explicación a este comportamiento podría ser que el pico de producción de leche se alcanzó sustentándose en la energía movilizada durante el inicio de la lactancia, cuando la vaca está en proceso de movilizar reservas de grasa subcutánea para apoyar las necesidades energéticas para la lactación (NRC 2001). Es probable que tanto los resultados en producción láctea al pico como, las concentraciones de $\beta$ HBA a los $30 \pm 3$ días posparto, respondan a la movilización de reservas corporales, puesto que aquellos animales con concentraciones mayores a 1,4 mmol. $\mathrm{l}^{-1} \beta \mathrm{HBA}$ a los $30 \pm 3$ días de lactancia fueron los que perdieron mayor condición corporal previo al parto $(\mathrm{p}<0,05)$. Lo cual indica mayor movilización de reservas que pudieron haber generado una mayor concentración de $\beta \mathrm{HBA}$ a los $30 \pm 3$ días posparto y a su vez dichas reservas fueron utilizadas como fuente generadora de precursores para la síntesis de la mayor producción durante el segundo mes de lactancia (producción pico), creando las diferencias $(\mathrm{p}<0,05)$ para esta variable productiva, entre animales con y sin el padecimiento. Buckley et ál. (2000) afirman que en el inicio de la lactación las vacas con alto mérito genético en producción tuvieron una mayor pérdida en condición corporal, que indica un balance energético negativo mayor, que vacas con mérito genético inferior. Investigaciones relacionadas a una mayor producción de leche en vacas que movilizaron más reservas corporales han sido reportadas previamente (Pedron et ál. 1993).

Los hallazgos de este estudio podrían contribuir en la disminución de la prevalencia de cetosis tipo I y II, mediante la implementación del diagnóstico individual y prácticas nutricionales y de manejo a nivel de finca, considerando los factores asociados a la enfermedad determinados en este trabajo. Sin embargo, es necesario considerar que los alcances de esta investigación podrían estar circunscritos al hato en estudio, por lo que sugerimos validar los alcances de esta investigación en otros hatos de ganado lechero cuya alimentación se sustente en el consumo de forrajes tropicales. 


\section{CONCLUSIONES}

La prevalencia y el grado de cetosis tipo I fueron mayores que la tipo II. El aumento en la pérdida de condición corporal preparto, duración del periodo seco y número de partos fueron factores asociados al desarrollo de cetosis, por lo que se recomienda sean tomados en consideración en futuras investigaciones. La medición de la condición corporal en el periodo preparto podría servir como un indicador para predecir la propensión de los animales a sufrir cetosis tipo I. Se recomienda el uso de medidores cuantitativos electroquímicos de $\beta \mathrm{HBA}$ sanguíneo, para un diagnóstico de la enfermedad más preciso. Con base en la información obtenida en esta investigación se sugiere realizar prácticas de manejo tendientes a evitar períodos secos superiores a 60 días, además la implementación de prácticas de alimentación para reducir la pérdida de condición corporal preparto en vacas multíparas y de alta producción, a fin de reducir la incidencia de esta enfermedad metabólica.

\section{AGRADECIMIENTOS}

Los autores expresan su agradecimiento al Ing. Agr. José Joaquín Jiménez Zamora, propietario de la finca Pasquí por facilitar el hato donde se realizó la investigación. Así como a los señores Jorge Enrique Volio Elbrech, Médico Veterinario Carlos Luis Madriz Bonilla, Javier Masis Calvo, Ing. Agr. Adrián Martínez Machado y Felipe Campos Arguedas por la valiosa colaboración brindada durante la realización de este estudio. De igual forma al Centro Regional de Informática para la Producción Animal Sostenible (CRIPAS) por suministrar el software de lectura VAMPP Bovino 3.0.

\section{LITERATURA CITADA}

ANDERSSON L. 1988. Subclinical ketosis in dairy cows. Metabolic diseases of ruminant livestock. Vet. Clin. North Am. Food Anim. Pract. 4:233-251.
AOAC. Association of Official Agricultural Chemists. 2000. Official methods of analysis. $16^{\text {th }}$ ed. Washington, D. C.

BAIRD D.G. 1982. Primary ketosis in the high producing dairy cow: clinical and subclinical disorders, treatment, prevention and outlook. J. Dairy Sci. 65:1-10.

BOBE G., YOUNG J.W., BELTZ D.C. 2004. Invited review: pathology, etiology, prevention, and treatment of fatty liver in dairy cows. J. Dairy Sci. 87:3105-3124.

BUCKLEY F., DILLON P., CROSSE S., FLYNN F., RATH M. 2000. The performance of Holstein Friesian dairy cows of high and medium genetic merit of milk production on grass-based feeding systems. Livestock Production Science. 64:107-119.

COPPOCK C.E., EVERETT R.W., NATZKE R.P., AINSLIE H.R. 1974. Effect of dry period length on Holstein milk production and selected disorders at parturition. J. Dairy Sci. 57:712-718.

DRACKLEY J.K. 1997. Minimizing ketosis in high producing dairy cows, pp. 63-82. In: Tri-State Dairy Nutrition Conference. Fort Wayne, Indiana.

DUFFIELD T.F. 2000. Subclinical ketosis in lactating dairy cattle: Metabolic disorders of ruminants. Vet. Clin. North Am. Food Anim. Pract. 16:231-253.

EPPERSON W.B. 2005. Risk factors for metabolic disease, pp. 31-43. In: Tri-State Dairy Nutrition Conference. Fort Wayne.

FERGUSON J.D., GALLIGAN D.T., THOMSEN N. 1994. Principal descriptors of body condition score in Holstein cows. J. Dairy Sci. 77:2695-2703.

FRENCH P.D. 2006. Dry matter intake and blood parameters of non lactating Holstein and Jersey cows in late gestation. J. Dairy Sci. 89:1057-1061.

GEARHART M.A., CURTIS C.R., ERB H.N., SMITH R.D., SNIFFEN C.J., CHASE L.E., COOPER M.D. 1990. Relationship of changes in condition score to cow health in Holsteins. J. Dairy Sci. 73:3132-3140.

GILLUND P., REKSEN O., GROHN Y.T. KARLBERG K. 2001. Body condition related to ketosis and reproductive performance in Norwegian dairy cows. J. Dairy Sci. 84:1390-1396.

GRUMMER R.R. 1993. Etiology of lipid-related metabolic disorders in periparturient dairy cows. J. Dairy Sci. 76:3882-3896.

HAYIRLI A., GRUMMER R.R., NORDHEIM E.V., CRUMP P.M. 2002. Animal and dietary factors affecting feed intake during the prefresh transition period in Holstein. J. Dairy Sci. 85:3430-3443.

INSTITUTO METEOROLÓGICO NACIONAL (IMN). 2012. Información meteorológica de la provincia de Cartago. San José, Costa Rica.

IWERSEN M., FALKENBERG U., VOIGTSBERGER R., FORDERUNG D., HEUWIESER W. 2009. Evaluation of an electronic cowside test to detect 
subclinical ketosis in dairy cows. J. Dairy Sci. 92:2618-2624.

JOHNSON D.G., OTTERBY D.E. 1981. Influence of dry period diet on early postpartum health, feed intake, milk production, and reproductive efficiency of Holstein cows. J. Dairy Sci. 64:290-295.

JUCHEM S.O., SANTOS F.A.P., IMAIZUMI H., PIRES A.V., BERNABÉ E.C. 2004. Production and blood parameters of Holstein cows treated prepartum with sodium monensin or propylene glycol. J. Dairy Sci. 87:680-689.

MACKLE T.R., PARR C.R., STAKELUM G.K., BRYANT A.M., MACMILLAN K.L. 1996. Feed conversion efficiency, daily pasture intake, and milk production of primiparous Fresian and Jersey cows calved at two different live weights. N.Z.J. Agric. Res. 39:357370.

NATIONAL RESEARCH COUNCIL. 2001. Nutrient Requirements of Dairy Cattle. $7^{\text {th }}$ rev. ed. National Academy Press. Washington, D.C. 381 p.

NIELSEN N.I., FRIGGENS N.C., CHAGUNDA G.G., INGVARTSEN K.L. 2005. Predicting risk of ketosis in dairy cows using in-line measurements of $\beta$-hidroxibutyrate: a biological model. J. Dairy Sci. 88:2441-2453.

OETZEL G. 2007. Herd-Level Ketosis-Diagnosis and Risk Factors. American Association of Bovine Practitioners $40^{\text {th }}$ Annual Conference, September 19, 2007-Vancouver, BC, Canada pp. 67-91.
OETZEL G., McGUIRK S. 2010. Fact Sheet-Cowside Blood BHBA Testing with a Hand-Held "Ketometer". University of Wisconsin-Madison, School of Veterinary Medicine. Madison, Wisconsin, U.S.A. Consultado 30-8-2010, disponible en http://www. vetmed.wisc.edu/dms/fapm/fapmtools/tci/BHBATesting-Update-090305.pdf

PEDRON O., CHELI F., SENATORE E., BAROLI D., RIZZI R. 1993. Effect of body condition score at calving on performance, some blood parameters, and milk fat acid composition in dairy cows. J. Dairy Sci. 76:2528-2535.

PRENDIVILLE R., PIERCE K.M., BUCKLEY F. 2009. An evaluation of production efficiencies among lactating Holstein-Friesian, Jersey, and Jersey $\mathrm{x}$ Holstein Friesian cows at pasture. J. Dairy Sci. 92:6176-6185.

TREACHER R.J., REID I.M., ROBERTS C.J. 1986. Effect of body condition at calving on the health and performance of dairy cows. Anim. Prod. 43:1-6.

VAN SOEST P., ROBERTSON J., LEWIS B. 1991. Methods for dietary fiber, neutral detergent fiber and nonstarch polysaccharides in relation to animal nutrition. J. Dairy Sci. 74:3586-3597.

WATTERS R.D., GUENTHER J.N., BRICKNER A.E., RASTANI R.R., CRUMP P.M., CLARK P.W., GRUMMER R.R. 2008. Effects of dry period length on milk production and health on dairy cattle. J. Dairy Sci. 91:2595-2603.

Todos los derechos reservados. Universidad de Costa Rica. Este artículo se encuentra licenciado con Creative Commons Reconocimiento-NoComercial-SinObraDerivada 3.0 Costa Rica. Para mayor información escribir a rac.cia@ucr.ac.cr 
